\title{
基于变径边界样本界面检测器的异常度检测方法
}

\author{
李 栋 $^{1}$ 孙 欣 ${ }^{2}$ 刘树林 ${ }^{2}$ \\ (1. 常州大学石油工程学院 常州 213164; \\ 2. 上海大学机电工程与自动化学院 上海 200072)
}

\begin{abstract}
摘要: 针对机械设备缺少故障样本时如何高效检测其异常度问题, 在分析训练样本的分布情况以及其在自己空间边界状态的 基础上, 优化训练样本半径, 提出一种变径边界样本界面检测器。利用变径边界样本界面检测器异常检测方法分析了轴承状 态数据, 不仅能反映出轴承的各种故障状态, 而且能通过异常度函数反映出故障的轻重程度。变径边界样本界面检测器的设 备异常度检测方法, 是在学习设备正常运行数据的基础上, 优化训练样本半径, 利用边界样本及其方位信息, 构建界面检测 器, 对设备的运行状态进行检测。变径边界样本界面检测器在构建过程中不需要使用设备的故障数据, 适用于对缺少故障数 据的机械设备进行异常状态检测。
\end{abstract}

关键词: 反面选择机理; 人工免疫算法; 异常度; 异常度检测; 轴承

中图分类号: TP306

\section{Equipment Abnormal Degree Detection Approach Based on Interface Detector with Variable-sized Boundary Samples}

\author{
LI Dong ${ }^{1} \quad$ SUN Xin $^{2} \quad$ LIU Shulin ${ }^{2}$ \\ (1. School of Petroleum Engineering, Changzhou University, Changzhou 213164; \\ 2. School of Mechatronics Engineering and Automation, Shanghai University, Shanghai 200072)
}

\begin{abstract}
The interface detector with variable-sized boundary samples (VI-detector) is presented based on the optimized radii of training samples according to their distributions and the place in the self space, which can detect the abnormal degree of equipment rapidly without fault sample. VI-detector not only reflects the various fault states of bearing, but also reflects the fault degree of the equipment by the abnormal degree function when analyzed the bearing state data used VI-detector. The VI-detector can detect the faults of equipment by learning normal data without fault data, which is built with variable-sized boundary samples and their position information. It can efficiently detect the abnormal of the equipment that lacks fault data.
\end{abstract}

Key words: negative selection algorithm; artificial immune algorithm; abnormal degree; anomaly detection; bearing

\section{0 前言}

设备异常检测与故障诊断技术是现代化机械设 备安全可靠运行的重要保障。由于机械设备不易 “带 病” 工作, 从而导致反映设备正常运行状态的数据 容易获取, 反映设备故障状态的数据获取困难。因 此, 在设备缺乏故障数据的情况下, 利用采集到的 设备正常运行数据开展故障诊断, 对保障设备安全 可靠运行有着重要的意义 $义^{[1-2]}$ 。

人工免疫系统是在生物免疫系统相关智能机理 的启发下，通过模拟生物免疫系统的工作原理、基

* 国家自然科学基金资助项目(51575331)。20190505 收到初稿, 20191020 收到修改稿
本功能和基本模型来解决各类复杂工程问题的具有 连续学习能力的自适应系统 ${ }^{[3-4]}$ 。反面选择算法是人 工免疫系统的主要方法, 可在无先验知识的情况下, 通过学习有限数量的正常数据便能检测出无限数量 的异常数据。正是由于反面选择算法的这一独特的 性能, 该方法提出后迅速得到了国内外众多学者的 广泛关注，相继提出了大量的相关算法，并在计算 机安全以及设备异常检测与故障诊断等领域得到了 应用 ${ }^{[5-6]}$ 。

国内外的专家学者围绕着提高反面选择算法的 检测率、降低其误报警率、减少检测器数量、提升 检测效率进行了大量的研究工作, 取得了一系列研 究成果 ${ }^{[7-12]}$ 。然而, 传统反面选择算法的检测器是 随机生成的, 即使相同的训练样本, 每次生成的检 
测器数量、大小、位置不同, 这严重制约了反面选 择算法的发展。近年来, 上海大学刘树林教授课题 组提出了一系列非随机检测器反面选择算法, 检测 器数量、大小、位置只和训练样本有关, 与训练次 数无关 ${ }^{[13-16]}$ 。基于非随机检测器反面选择算法的这 一特性, 提出了小样本在线学习异常检测方法, 在 测试过程中利用已正确识别的样本逐步完善检测 器, 而不用重新训练, 实现了只用一个样本训练即 可完成异常检测 ${ }^{[15,17]}$ 。界面检测器利用自己空间的 边界样本及其方位信息进形异常检测, 是非随机检 测器反面选择算法中检测率最高的一类 ${ }^{[13-15]}$ 。然而, 相同条件下, 界面检测器的误报警率也是最高的。 因此, 如何在保证界面检测器高检测率的基础上, 进一步幅降低其误报警率, 具有重要的研究意义。

\section{1 界面检测器及其特点}

界面检测器(Interface detector, I-detector), 是一 个或多个紧紧围绕自己空间的超曲面, 可以由自己 空间最外层的样本, 即边界样本描述 ${ }^{[14-15]}$ 。

$$
D=\left\{<\boldsymbol{b}_{i}, r_{s}, p_{i}>\mid \boldsymbol{b}_{i} \in \boldsymbol{B}, r_{s} \in \mathbf{R}\right\}
$$

式中 $\boldsymbol{b}_{i}$ 一边界样本中心坐标;

$r_{s}$ 一自己样本半径;

$p_{i}$ 一边界样本 $\boldsymbol{b}_{i}$ 的方位信息;

$B$ 一一边界样本集。

显然, 在二维空间界面检测器是一条或多条封 闭的曲线, 如图 1 所示。自己样本和非己样本位于 界面检测器的两侧。

- 自己样本
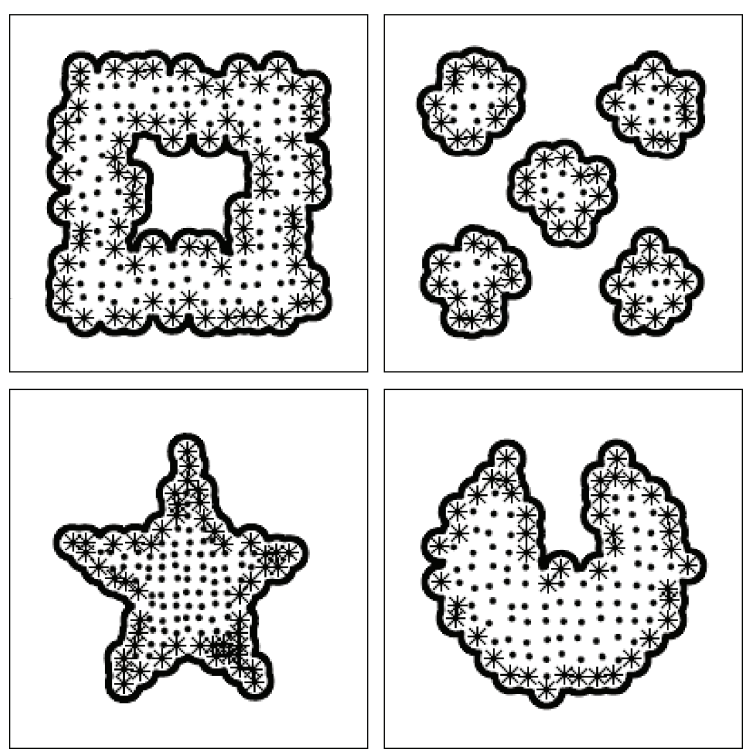

图 1 不同形状自己空间的界面检测器
由于界面检测器在训练时样本半径 $r_{s}$ 是固定不 变的, 从而导致边界样本的半径 $r_{s}$ 也是固定不变的。 当训练样本半径 $r_{s}$ 增大时, 由其构建的自己空间内 部孔洞体积减少，从而导致误报警率降低; 与此同 时, 训练样本覆盖到的非己空间也随之增多, 从而 使得检测率降低。当训练样本半径 $r_{s}$ 减小时, 由其 构建的自己空间内部孔洞体积增大，从而导致误报 警率升高; 与此同时, 训练样本覆盖到的非己空间 也随之减少, 从而使得检测率升高。由于界面检测器 在训练时所有训练样本的半径 $r_{s}$ 是相同的, 从而导致 界面检测器的检测率和误报警率这对矛盾无法调节。

\section{2 变径界面检测器}

\section{1 基本思想}

由界面检测器的特点可知, 其检测率与自己空 间覆盖到非己空间的多少有关，非己空间被自己空 间覆盖的越少检测率越高; 误报警率与自己空间内 部的孔洞有关，孔洞越多，误报警率越高。为了在 一定程度上缓解界面检测器的检测率与误报警率这 对矛盾, 希望靠近自己空间边界的样本半径 $r_{s}$ 相对 较小, 位于自己空间内部的样本半径 $r_{s}$ 相对较大。 在这种思想的启发, 提出了一种边界样本半径不是 固定值的界面检测器, 即变径边界样本界面检测器 (Interface detector with variable-sized boundary samples, VI-detector), 用于设备的异常检测。将其 描述为

$$
D=\left\{<\boldsymbol{b}_{i}, r_{i}, p_{i}>\mid \boldsymbol{b}_{i} \in \boldsymbol{B}, r_{i} \in \mathbf{R}\right\}
$$

式中 $\boldsymbol{b}_{i}$ 一边界样本中心坐标;

$r_{i}$ —边界样本 $\boldsymbol{b}_{i}$ 的半径;

$p_{i}$ 一边界样本 $\boldsymbol{b}_{i}$ 的方位信息。

变径边界样本界面检测器的核心工作是找到边 界样本并确定其对应的半径。边界样本是训练样本 的一个子集, 因此, 要确定边界样本的半径, 首先 需要在基准半径的基础上确定每个训练样本的半 径。每个训练样本的半径与所有训练样本在状态空 间的分布及其在状态空间所处的位置有关，因此在 确定每个训练样本的半径时需要综合考虑这两方面 的因素。

利用样本的分布密度确定其相应的半径是最常 用的方法之一, 密度高的地方样本半径相对较大, 密度小的地方样本半径相对较小。计算样本分布密 度的方法很多, 在此用样本之间的距离描述样本分 布密度, 并确定其半径。

$$
r_{i}=\delta_{i} r_{r e f}
$$


式中 $r_{i}$ 一训练样本 $t_{i}$ 的半径;

$r_{r e f}$ 一训练样本的基准半径;

$\delta_{i}$-一训练样本 $t_{i}$ 的半径调节权重。

$$
\delta_{i}=\frac{d_{\text {avg }}}{d_{i}}
$$

式中 $d_{a v g}$ 一训练样本之间的平均距离;

$d_{i}$ 一一训练样本 $t_{i}$ 到其他样本的平均距离。

$$
\begin{gathered}
d_{\text {avg }}=\frac{\sum_{i, j=1}^{n} d_{i j}}{n(n-1)}, i, j=1,2, \cdots, n, i \neq j \\
d_{i}=\frac{\sum_{j=1}^{n} d_{i j}}{n-1}, j=1,2, \cdots, n, i \neq j
\end{gathered}
$$

式中 $d_{i j}$ 一训练样本 $t_{i}$ 与 $t_{j}$ 之间的距离;

$n$-一训练样本的数量。

当样本整体相对集中时, 由式(1)确定样本半 径, 生成的自己空间内部孔洞较少, 如图 2a 所示; 但是当样本分布不是整体相对集中时, 由式(1)确定 样本半径, 生成的自己空间内部则有许多孔洞, 如 图 $2 b$ 所示。

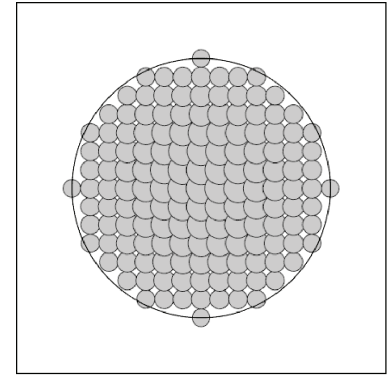

(a)

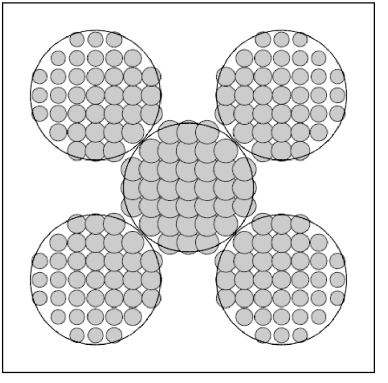

(b)
图 2 训练样本生成的自己空间

因此, 从四个方面对式(1)进行修正。首先, 式 (1)考虑的是样本 $t_{i}$ 与其他所有样本之间的分布关 系, 属于全局考虑。为了即体现样本 $t_{i}$ 与全局样本 之间的分布关系, 又体现样本 $t_{i}$ 与其周围局部样本 之间的分布关系, 利用 $t_{i}$ 到距离其最近的 $k$ 个样本 的平均距离对式(1)中的 $\delta_{i}$ 进行修正。

$$
\begin{gathered}
\tau_{i}=\frac{d_{\text {avg }}}{d_{i k}} \\
d_{i k}=\frac{\sum_{j=1}^{k} d_{i j}}{k}, j=1,2, \cdots, k, i \neq j
\end{gathered}
$$

式中 $\tau_{i}$ 一样本 $t_{i}$ 的样本分布系数;

$d_{i k}$ 一样本 $t_{i}$ 与其最近 $k$ 个样本的平均距离;

$k$ 一一距离 $t_{i}$ 最近的 $k$ 个样本。

由式(6)可以看出, 当 $k=n$ 时, $\delta_{i}=\tau_{i}$ 。

其次, 不仅考虑样本 $t_{i}$ 与其他样本之间的分布
关系, 还要考虑该样本 $t_{i}$ 在自己空间的位置。样本 $t_{i}$ 在自己空间的不同位置赋予不同的数值, 例如在 二维空间, 样本 $t_{i}$ 会存在五种可能的状态: 不是边 界样本、三侧有自己样本的边界样本、两侧有自己 样本的边界样本、一侧有自己样本的边界样本和周 围没有自己样本的边界样本, 这五种状态分别取值 为 $0.4 、 0.3 、 0.2 、 0.1 、 0$ 。在高维空间, 需要根据 实际情况进行调整。

由于界面检测器在确定边界样本时用到参数 $r_{s}$, 且 $r_{s}$ 是一个经验值 ${ }^{[13-15]}$ 。由于无法直接确定每 个样本在空间的位置, 为了较为全面的分析样本在 空间的分布情况, 考虑不同参数情况下样本在自己 空间的位置。论文中考虑了五种条件下样本在自己 空间的位置, 即 $0.75 r_{r e f} 、 r_{r e f} 、 1.25 r_{r e f} 、 1.5 r_{r e f} 、 2 r_{r e f}$, 记为 $w_{1} 、 w_{2} 、 w_{3} 、 w_{4}$ 和 $w_{5}$, 并分别赋予权值 0.3 、

$0.25 、 0.2 、 0.15$ 和 0.1 , 加权求和得到样本 $t_{i}$ 在自己 空间的位置系数 $w_{i}$ 。

再者, 考虑到 $\tau_{i}$ 与 $w_{i}$ 的乘积可能过大引起训练 样本半径不可控, 将其乘积利用式(7)归一化

$$
\lambda_{i}=1+2 \times \frac{\sigma_{i}-\min (\sigma)}{\max (\sigma)-\min (\sigma)}
$$

式中 $\lambda_{i}$ 一训练样本 $t_{i}$ 的半径调节系数;

$\sigma_{i}$ 一训练样本的 $\tau_{i}$ 与 $w_{i}$ 乘积。

此时, 训练样本的半径可用式(8)计算

$$
r_{i}=\lambda_{i} r_{\text {ref }}
$$

由于边界样本的半径半径过大, 覆盖到非己空 间的面积增加, 导致检测率降低。为此, 在式(8)的 基础上增加一个调节参数 $\mu$, 其取值为 $[0.5,1]$, 则 训练样本的半径的计算公式修正为

$$
r_{i}=\mu \lambda_{i} r_{r e f}
$$

根据式(9)修正后的图 $2 b$ 如图 3 所示, 两图的 基准半径相同, $r_{r e f}=0.025$ 。从图 3 可以看出, 修正 后的训练样本形成的自己空间覆盖到的非已空间较 少, 且内部孔洞也较少, 因此与真实自己空间的匹 配度较高。

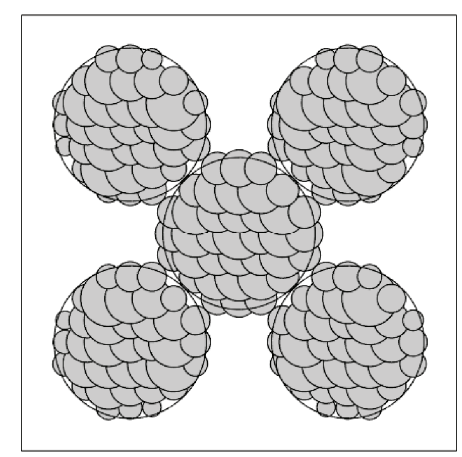

图 3 修正后训练样本生成的自己空间 $(k=12, \mu=0.85)$ 


\section{2 变径界面检测器的训练与测试过程}

VI-detector 的训练过程是在界面检测器训练过 程的基础上补充了计算训练样本半径调节系数部 分。VI-detector 训练过程的主要步骤如下。

(1) 输入参数 $r_{r e f} 、 k$ 和 $\mu$ 。

(2) 计算训练样本的样本分布系数 $\tau_{i}$ 。

(3) 寻找边界样本, 解码其方位信息, 计算 $w_{i}$ 。

(4) 计算训练样本的半径调节系数 $\lambda_{i}$ 。

(5) 计算训练样本调整后的半径 $r_{i}$ 。

(5) 利用 $\min \left(r_{i}\right)$ 确定边界样本并编码其方位 信息。

(6) 输出 VI-detector 相关信息。

VI-detector 的测试过程与界面检测器测试过程 相同。

\section{3 仿真与实例分析}

\section{1 二维人造数据集仿真分析}

用图 4 所示的二维人造数据集对 VI-detector 在 异常检测方面的性能进行了验证, 并与 I-detector 的异常检测性能进行对比。

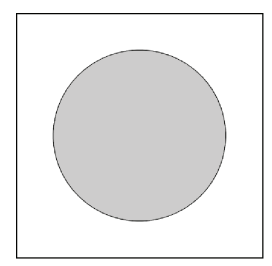

(a) 圆形

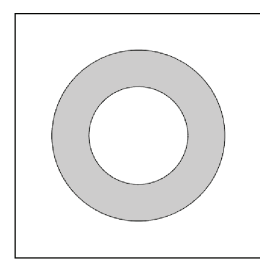

(b) 圆环
图 4 仿真试验自己空间形状

在状态空间 $[0,1]^{2}$ 生成 1639 个样本, 其中图 $4 \mathrm{a}$ 有自己样本 609 个, 图 4b 有自己样本 411 个。随机 选取 200 个自己样本作为训练样本, 随机选取 800 个非已样本, 从剩余的自己样本中随机选取 200 个 作为测试样本。重复计算 100 次取平均值作为仿真 试验结果。

图 5 为参数 $k$ 对 VI-detector 异常检测性能影响 的仿真试验结果图。为了方便对比, 将相同条件下 I-detector 的检测率和误报警率标记在图中横坐标 0 处; VI-detector 的检测率是其误报警率与 I-detector 的误报警率相近时对应的检测率值; VI-detector 的 误报警率是其检测率与 I-detector 的检测率相近时 对应的误报警率值。

从图 5 中可以看出, 随着参数 $k$ 的增大, VI-detector 的检测率有波动, 但整体呈上升趋势, 但是上升的幅度很小, 可以忽略; VI-detector 的误 报警率整体呈下降趋势, 但是降低的幅度较小。当 $k<6$ 的时候, VI-detector 的误报警率波动较大, 这

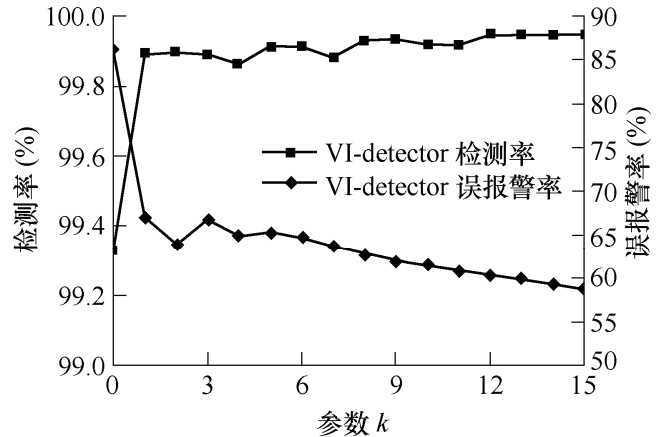

(a) 圆形数据仿真结果

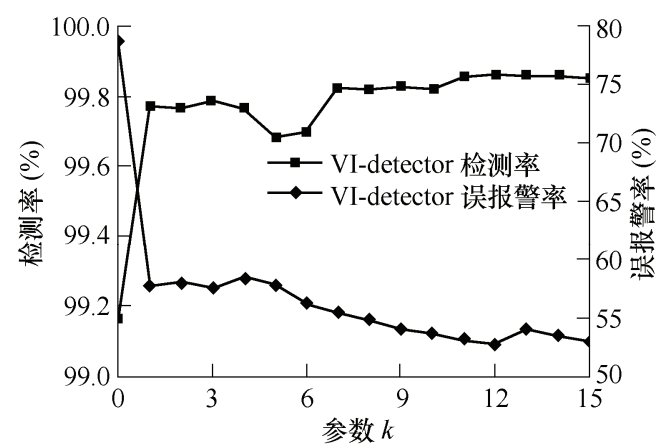

(b) 圆环数据仿真结果

图 5 参数 $k$ 对仿真试验结果的影响 $\left(r_{r e f}=0.02\right)$

是由于参数 $k$ 较小的时候, 过于考虑样本在局部分 布情况，不能很好地体现样本在自己空间的分布状 态; 当参数 $k$ 过大的时候, 过于考虑样本在自己空 间的分布情况，不能很好地体现样本在局部的分布 状态。因此, 要根据样本的分布情况合理的选择参 数 $k$ 。在后续的二维数据集仿真中，参数 $k$ 取 12 。

图 6 为参数 $\mu$ 对 VI-detector 异常检测性能影响 的仿真试验结果图, 将相同条件下 I-detector 的检测 率和误报警率标记在图中的箭头处。

从图 6 可以看出, 随着参数 $\mu$ 的增加, VI-detector 的检测率和误报警率下降，这是由于参 数 $\mu$ 取值较小时, 边界样本覆盖到的非已空间较少, 导致检测率较高, 但是在自己空间内部, 尤其是靠 近自己空间边界的地方形成许多孔洞，从而导致误 报警率较高; 参数 $\mu$ 取值较大时, 边界样本覆盖到 的非已空间较多，导致检测率较低，但是在自己空 间内部，孔洞减少，从而导致误报警率较低。然而， 参数 $\mu$ 存在一个合理的取值范围, 此时 VI-detector 的检测率比 I-detector 的检测率高, VI-detector 的误 报警率比 I-detector 的误报警率低。

图 7 为 VI-detector 和 I-detector 随样本基准半径

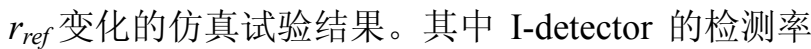
和误报警率直接使用仿真结果; VI-detector 的检测 率是其误报警率与 I-detector 的误报警率相近，且小 于 I-detector 误报警率的取值，VI-detector 的误报警 率是其检测率与 I-detector 的检测率相近, 且大于 I-detector 检测率的取值。 


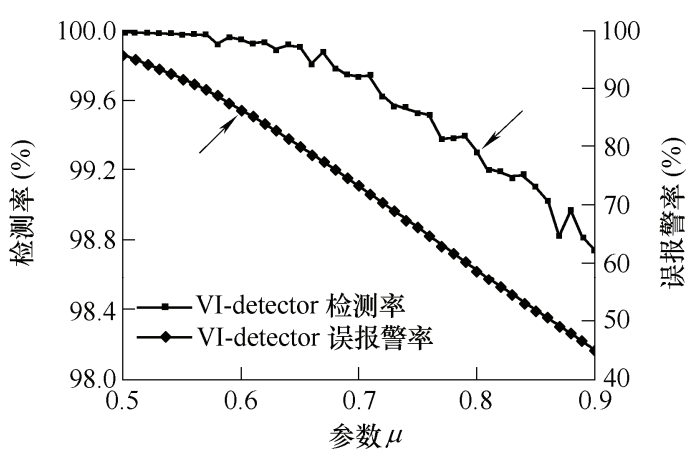

(a) 圆形数据仿真结果

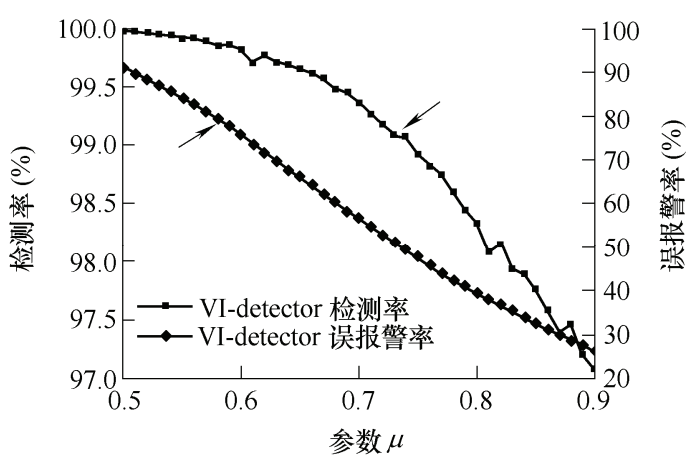

(b) 圆环数据仿真结果

图 6 参数 $\mu$ 对仿真试验结果的影响 $\left(r_{r e f}=0.02, k=12\right)$

从图 7 中可以看出, 通过式(8)调整训练样本的 半径, 可以保证在 VI-detector 的检测率不低于 I-detector 检测率的同时, 有效降低误报警率; 或者 在保证在 VI-detector 的误报警率不高于 I-detector 误报警率的同时，有效提高检测率。

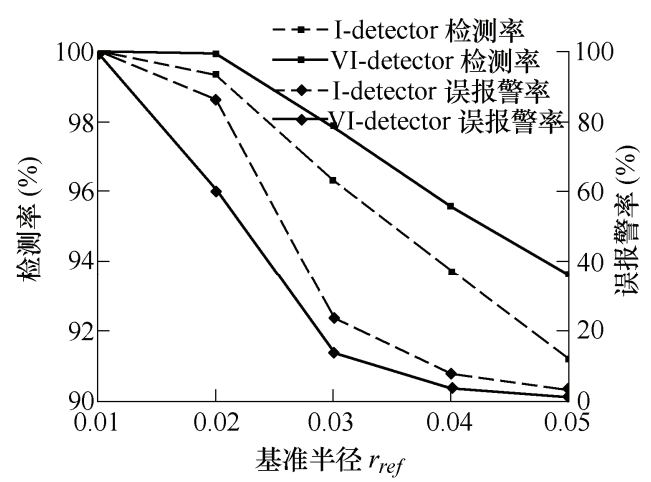

(a) 圆形数据仿真结果

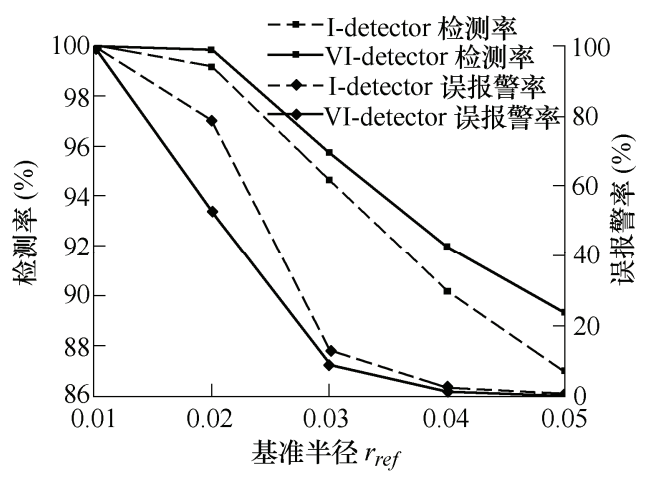

(b) 圆环数据仿真结果

图 7 仿真试验结果图 $(k=12)$

\subsection{Iris 数据仿真分析}

用 UCI 标准数据库中的 Iris 数据集进一步验证 VI-detector 在异常检测方面的性能, 并与文献中异 常检测方法的异常检测性能进行对比。

作为模式识别领域使用最广泛的的数据集之 一, Iris 数据集共有三类 150 个样本。将数据归一 化至 $[0,1]^{4}$, 其中一类样本作为正常样本, 其余两类 作为异常样本, 正常样本 $100 \%$ 或随机选取 $50 \%$ 作 为训练样本, 其余全部作为测试样本。

VI-detector 与其他几种异常检测方法的异常检 测结果如表 1 所示, 表 1 中的其他几种异常检测方 法的数据来自文献[12-14]和文献[18]。VI-detector 设定 Setosa、Versicolour 和 Virginica 的 $k$ 值分别是 $10 、 1$ 和 $4, \mu$ 值分别是 $1 、 0.58$ 和 0.5 。

表 1 中当 $50 \%$ 的正常样本训练时, VI-detector、 约简边界样本的界面检测器 (Interface detector with reduction boundary samples, RI-detector)和 I-detector 的相关数据是重复计算 100 次取平均值 的结果; 当正常样本全部作为训练样本时,

VI-detector、RI-detector 和 I-detector 的检测率 和检测器数量只需要计算一次, 这是由于 VI-detector、RI-detector 和 I-detector 的检测器是非 随机生成的, 当训练样本一定时, 生成的检测器大 小、数量和位置固定不变, 从而计算结果也不会 变化。

从表 1 中可以看出, 与其他几种异常检测方法 相比, VI-detector 的检测率最高, 且有效降低了 RI-detector 和 I-detector 的误报警率, 是一种具有高 检测率和较高误报警率的异常检测方法。

\section{3 轴承故障数据分析}

采用凯斯西储大学轴承故障数据进一步验证 VI-detector 的异常检测性能。用 $\mathrm{db} 16$ 小波包 8 层分解风扇端加速度采样数据, 特征向量选用高 频小波系数能量。生成 500 个正常样本, 每类故 障 50 个样本。为提高运行效率, 用 PCA 将数据 降维, 并将其归一化至 $[0,1]^{4}$ 。根据 VI-detector 的特性将文献[14]中的异常度函数修正为

$$
\mu_{A}(t)= \begin{cases}0 & \boldsymbol{t} \in S \\ \frac{2}{\pi} \arctan \left(d-r_{i}\right) & \boldsymbol{t}>N\end{cases}
$$

式中 $t$ 一测试样本;

$d-\boldsymbol{t}$ 至边界样本 $\boldsymbol{b}$ 距离的最小值;

$r_{i}$ 一距离 $\boldsymbol{t}$ 最近边界样本的半径。 
表 1 不同异常检测方法对 Iris 数据集的检测情况

\begin{tabular}{|c|c|c|c|c|c|c|c|}
\hline \multirow{2}{*}{ 训练数据 } & \multirow{2}{*}{ 所用算法 } & \multicolumn{2}{|c|}{ 检测率(\%) } & \multicolumn{2}{|c|}{ 误报警率(\%) } & \multicolumn{2}{|c|}{ 检测器数量 } \\
\hline & & 平均值 & 标准差 & 平均值 & 标准差 & 平均值 & 标准差 \\
\hline \multirow{9}{*}{$\begin{array}{c}\text { Setosa } \\
100 \%\end{array}$} & MILA & 95.16 & 1.79 & 0 & 0 & 1000 & 0 \\
\hline & NSA & 100 & 0 & 0 & 0 & 1000 & 0 \\
\hline & V-detector & 99.98 & 0.14 & 0 & 0 & 20 & 7.87 \\
\hline & ocSVM & 100 & 0 & 4 & 0 & - & - \\
\hline & Self-detector & 100 & 0 & 0 & 0 & 50 & 0 \\
\hline & VTI-detector & 100 & 0 & 0 & 0 & - & - \\
\hline & I-detector & 100 & 0 & 0 & 0 & 50 & 0 \\
\hline & RI-detector & 100 & 0 & 0 & 0 & 37 & 0 \\
\hline & VI-detector & 100 & 0 & 0 & 0 & 50 & 0 \\
\hline \multirow{9}{*}{$\begin{array}{c}\text { Setosa } \\
50 \%\end{array}$} & MILA & 94.02 & 2.44 & 8.42 & 1.56 & 1000 & 0 \\
\hline & NSA & 100 & 0 & 11.18 & 2.17 & 1000 & 0 \\
\hline & V-detector & 99.97 & 0.17 & 1.32 & 0.95 & 16.44 & 5.63 \\
\hline & ocSVM & 100 & 0 & 5.56 & 4.88 & - & - \\
\hline & Self-detector & 100 & 0 & 9.98 & 3.18 & 25 & 0 \\
\hline & VTI-detector & 100 & 0 & 0.22 & 0.69 & - & - \\
\hline & I-detector & 100 & 0 & 18.8 & 6.27 & 25 & 0 \\
\hline & RI-detector & 100 & 0 & 20.32 & 6.11 & 19.83 & 1.38 \\
\hline & VI-detector & 100 & 0 & 4.72 & 3.3 & 25 & 0 \\
\hline \multirow{9}{*}{$\begin{array}{l}\text { Versicolour } \\
100 \%\end{array}$} & MILA & 84.37 & 2.79 & 0 & 0 & 1000 & 0 \\
\hline & NSA & 95.67 & 0.69 & 0 & 0 & 1000 & 0 \\
\hline & V-detector & 85.95 & 2.44 & 0 & 0 & 153.24 & 38.8 \\
\hline & ocSVM & 90 & 0 & 8.38 & 1.60 & - & - \\
\hline & Self-detector & 98 & 0 & 0 & 0 & 50 & 0 \\
\hline & VTI-detector & 91 & 0 & 0 & 0 & - & - \\
\hline & I-detector & 98 & 0 & 0 & 0 & 50 & 0 \\
\hline & RI-detector & 98 & 0 & 0 & 0 & 44 & 0 \\
\hline & VI-detector & 99 & 0 & 0 & 0 & 50 & 0 \\
\hline \multirow{9}{*}{$\begin{array}{c}\text { Versicolour } \\
50 \%\end{array}$} & MILA & 84.46 & 2.70 & 19.60 & 2.00 & 1000 & 0 \\
\hline & NSA & 96 & 0.45 & 22.2 & 1.25 & 1000 & 0 \\
\hline & V-detector & 88.30 & 2.77 & 8.42 & 2.12 & 110.08 & 22.61 \\
\hline & ocSVM & 90.57 & 2.92 & 7.72 & 3.84 & - & - \\
\hline & Self-detector & 99.03 & 0.99 & 15.72 & 3.55 & 25 & 0 \\
\hline & VTI-detector & 96.33 & 2.18 & 10.38 & 3.65 & - & - \\
\hline & I-detector & 99.04 & 1.0 & 30.36 & 7.59 & 25 & 0 \\
\hline & RI-detector & 99.04 & 1.0 & 31.88 & 8.15 & 23.06 & 1.09 \\
\hline & VI-detector & 99.1 & 9.0 & 26.88 & 1.03 & 25 & 0 \\
\hline \multirow{9}{*}{$\begin{array}{c}\text { Virginica } \\
100 \%\end{array}$} & MILA & 75.75 & 2.01 & 0 & 0 & 1000 & 0 \\
\hline & NSA & 92.51 & 0.74 & 0 & 0 & 1000 & 0 \\
\hline & V-detector & 81.87 & 2.78 & 0 & 0 & 218.36 & 66.11 \\
\hline & ocSVM & 75 & 0 & 5.28 & 0.96 & - & - \\
\hline & Self-detector & 99 & 0 & 0 & 0 & 50 & 0 \\
\hline & VTI-detector & 94 & 0 & 0 & 0 & - & - \\
\hline & I-detector & 93 & 0 & 0 & 0 & 50 & 0 \\
\hline & RI-detector & 91 & 0 & 0 & 0 & 46 & 0 \\
\hline & VI-detector & 100 & 0 & 0 & 0 & 50 & 0 \\
\hline \multirow{9}{*}{$\begin{array}{c}\text { Virginica } \\
50 \%\end{array}$} & MILA & 88.96 & 2.04 & 24.98 & 2.56 & 1000 & 0 \\
\hline & NSA & 97.18 & 0.71 & 33.26 & 0.96 & 1000 & 0 \\
\hline & V-detector & 93.58 & 2.33 & 13.18 & 3.24 & 108.12 & 30.74 \\
\hline & ocSVM & 78.42 & 8.35 & 9.36 & 4.04 & - & - \\
\hline & Self-detector & 99.09 & 0.30 & 26.36 & 4.23 & 25 & 0 \\
\hline & VTI-detector & 96.8 & 1.81 & 8.30 & 4.60 & - & - \\
\hline & I-detector & 99.28 & 0.42 & 51.32 & 8.66 & 25 & 0 \\
\hline & RI-detector & 99.23 & 0.45 & 51.44 & 9.24 & 23.97 & 0.887 \\
\hline & VI-detector & 99.42 & 0.64 & 50.16 & 9.9 & 25 & 0 \\
\hline
\end{tabular}

随机取 450 个正常样本作为训练样本, 剩余样 本作为测试样本, 参数 $r_{r e f}=0.15, k=10, \mu=1$, 重复 计算 100 次取平均值的作为仿真试验结果。仿真试 验结果如图 8 所示, 各类样本的异常度分布统计结 果如表 2 所示。

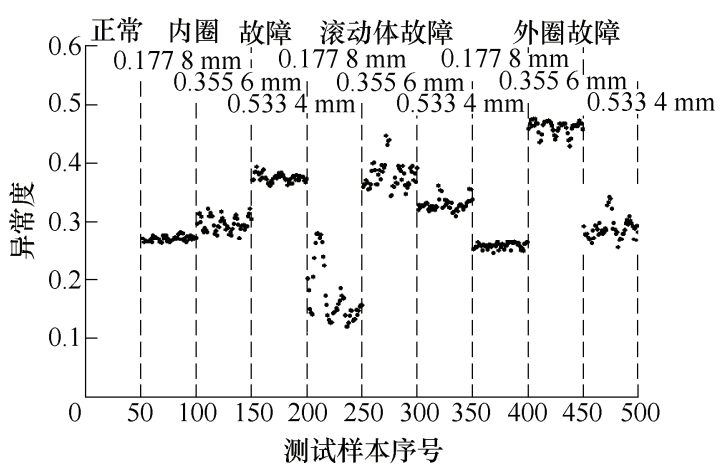

图 8 各类样本异常度分布图

表 2 各类样本异常度分布统计表

\begin{tabular}{|c|c|c|c|c|c|}
\hline \multicolumn{2}{|c|}{ 样本类型 } & \multirow{2}{*}{$\begin{array}{c}\text { 最小异常度 } \\
0 \\
\end{array}$} & \multirow{2}{*}{$\begin{array}{c}\text { 最大异常度 } \\
0\end{array}$} & \multicolumn{2}{|c|}{$\begin{array}{c}\text { 平均异常度 } \\
\text { (标准差) }\end{array}$} \\
\hline 正常 & & & & 0 & (0) \\
\hline \multirow{3}{*}{$\begin{array}{l}\text { 内圈故障 } \\
\text { 深度/mm }\end{array}$} & 0.1778 & 0.2631 & 0.2826 & 0.2705 & $(0.0047)$ \\
\hline & 0.3556 & 0.2716 & 0.3227 & 0.2969 & $(0.0127)$ \\
\hline & 0.5334 & 0.3616 & 0.3942 & 0.3751 & $(0.0067)$ \\
\hline \multirow{3}{*}{$\begin{array}{l}\text { 滚动体故障 } \\
\text { 深度/mm }\end{array}$} & 0.1778 & 0.1189 & 0.2801 & 0.1716 & $(0.0492)$ \\
\hline & 0.3556 & 0.3439 & 0.4474 & 0.3801 & $(0.0230)$ \\
\hline & 0.5334 & 0.3093 & 0.3618 & 0.3294 & $(0.0108)$ \\
\hline \multirow{3}{*}{$\begin{array}{l}\text { 外圈故障 } \\
\text { 深度/mm }\end{array}$} & 0.1778 & 0.2449 & 0.2661 & 0.2580 & $(0.0050)$ \\
\hline & 0.3556 & 0.4293 & 0.4750 & 0.4609 & (0.011 0) \\
\hline & 0.5334 & 0.2555 & 0.3423 & 0.2884 & $(0.0186)$ \\
\hline
\end{tabular}

从表 2 和图 8 中可以看出, VI-detector 的检测率 为 $100 \%$, 误报警率为 0 , 而且能有效反映出不同类型 故障, 以及不同故障程度的同类故障的异常度分布。

\section{4 结论}

(1) VI-detector 是在全面考虑样本之间的分布 情况以及边界样本在自己空间边界的具体类型基础 上提出的异常检测方法, 能高效地进行正常/异常识 别, 可以用于设备异常状态检测。

(2) 通过两组人造数据集、UCI 标准数据库中 Iris 数据集和凯斯西储大学轴承故障数据的仿真试 验发现, 相同条件下 VI-detector 的检测率更高, 其 误报警率比 RI-detector 和 I-detector 的误报警率有显 著降低。

(3) VI-detector 的检测效果受训练样本基准半 径 $r_{r e f}$ 以及参数 $k$ 和 $\mu$ 取值的影响, 目前这些参数的 取值主要依靠经验, 需要进一步研究参数自适应优 化方法。 
(4) VI-detector 属于单分类方法, 能通过异常度 函数有效反映各类故障, 然而, 异常度对设备故障 的评判比较单一, 需要进一步研究基于 VI-detector 的多分类方法, 将其推广应用于设备故障诊断。

\section{参 考 文 献}

[1] 雷亚国, 贾峰, 孔德同, 等. 大数据下机械智能故障诊 断的机遇与挑战 [J]. 机械工程学报, 2018, 54(5): 94-104.

LEI Yaguo, JIA Feng, KONG Detong, et al. Opportunities and challenges of machinery intelligent fault diagnosis in big data era[J]. Journal of Mechanical Engineering, 2018, 54(5): 94-104.

[2] SHAO Siyu, SUN Wenjun, YAN Ruqiang, et al. A deep learning approach for fault diagnosis of induction motors in manufacturing[J]. Chinese Journal of Mechanical Engineering, 2017, 30(6): 1347-1356.

[3] BAYAR N, DARMOUL S, HAIJRI-GABOUJ S. Fault detection, diagnosis and recovery using artificial immune systems: A review[J]. Engineering Application of Artificial Intelligence, 2015, 46: 43-57.

[4] DASGUPTA D, YU S, NINO F. Recent advances in artificial immune systems: models and applications[J]. Applied Soft Computing, 2011, 11(2): 1574-1587.

[5] FORREST S, PERELSON A S, ALLEN L, et al. Self-nonself discrimination in a computer $[\mathrm{C}] / /$ Proceedings of 1994 IEEE Computer Society Symposium on Research in Security and Privacy, 16-18 May, 1994, Oakland, CA, USA. New York: IEEE, 1994: 202-212.

[6] 金章赞, 廖明宏, 肖刚. 否定选择算法综述 $[J]$. 通信学 报, 2013，34(1): 159-170.

JIN Zhangzan, LIAO Mingong, XIAO Gang. Survey of negative selection algorithms[J]. Journal on Communications, 2013, 34(1): 159-170.

[7] DASGUPTA D, GONZÁLEZ F. An immunity-based technique to characterize intrusions in computer networks[J]. IEEE Transactions on Evolutionary Computation, 2002, 6(3): 281-291.

[8] ZHOU Ji, DASGUPTA D. V-detector: An efficient negative selection algorithm with "probably adequate" detector coverage[J]. Information Sciences, 2009, 179(10): 1390-1406.

[9] ABID A, KHAN M T, DE SILVA C W. Layered and real-valued negative selection algorithm for fault detection[J]. IEEE Systems Journal, 2018, 12(3): 2960-2969.

[10] 刘树林, 黄文虎, 夏松波. 基于免疫机理的往复压缩机 气阀故障检测方法[J]. 机械工程学报, 2004, 40(7): 156-160.

LIU Shulin, HUANG Wenhu, XIA Songbo. Fault detection approach based on immune mechanism for gas valves of reciprocating compressors[J]. Chinese Journal of Mechanical Engineering, 2004, 40(7): 156-160.

[11] YANG Tao, CHEN Wen, LI Tao. An antigen space density based real-valued negative selection algorithm[J]. Applied Soft Computing, 2017, 61: 860-874.

[12] 张宏利, 刘树林, 缴文会, 等. 基于可变阈值信息检测 器的设备异常度检测方法[J]. 机械工程学报, 2013, 49(8): 25-31.

ZHANG Hongli, LIU Shulin, JIAO Wenhui, et al. Equipment abnormal degree detection approach based on variable threshold information detector[J]. Journal of Mechanical Engineering, 2013, 49(8): 25-31.

[13] 李栋, 刘树林, 刘颖慧, 等. 基于自适应超环检测器的 设备异常度检测方法 [J]. 机械工程学报, 2014, 50(12): 17-24.

LI Dong, LIU Shulin, LIU Yinghui, et al. Equipment abnormal degree detection approach based on adaptive hyper-ring detector[J]. Journal of Mechanical Engineering, 2014, 50(12): 17-24.

[14] 李栋, 刘树林, 张宏利. 基于约简边界样本界面检测器 的设备异常度检测方法 [J]. 机械工程学报, 2015, 51(16): 77-86.

LI Dong, LIU Shulin, ZHANG Hongli. Equipment abnormal degree detection approach based on Interface detector with reduction boundary samples[J]. Journal of Mechanical Engineering, 2015, 51(16): 77-86.

[15] LI Dong, LIU Shulin, ZHANG Hongli. A negative selection algorithm with online adaptive learning under small samples for anomaly detection[J]. Neurocomputing, 2015, 149: 515-525.

[16] LI Dong, LIU Shulin, ZHANG Hongli. Negative selection algorithm with constant detectors for anomaly detection[J]. Applied Soft Computing, 2015, 36: 618-632.

[17] LI Dong, LIU Shulin, ZHANG Hongli. A boundary-fixed negative selection algorithm with online adaptive learning under small samples for anomaly detection[J]. Engineering Applications of Artificial Intelligence, 2016, 50: 93-105.

[18] STIBOR T, MOHR P, TIMMIS J. Is negative selection appropriate for anomaly detection?[C]// Proceedings of the 7th Annual Conference on Genetic and Evolutionary Computation, 25-29 June, 2005, Washington DC, USA. New York: ACM, 2005: 321-328.

作者简介: 李楼(通信作者), 男, 1981 年出生, 博士, 副教授, 硕士研 究生导师。主要研究方向为设备智能故障诊断方法。

E-mail: lidong@cczu.edu.cn

孙欣, 女, 1994 年出生, 博士研究生。主要研究方向为设备智能故障诊 断方法。

E-mail: sunxin52@shu.edu.cn

刘树林, 男, 1963 年出生, 博士, 教授, 博士研究生导师。主要研究方 向为复杂设备故障诊断。

E-mail: 1s1346@shu.edu.cn 